\title{
Active Asteroids in the NEO Population
}

\author{
Peter Jenniskens \\ SETI Institute \\ 189 Bernardo Ave, Mountain View, CA 94043, USA \\ email: petrus.m.jenniskens@nasa.gov
}

\begin{abstract}
Some main-belt asteroids evolve into near-Earth objects. They can then experience the same meteoroid-producing phenomena as active asteroids in the main belt. If so, they would produce meteoroid streams, some of which evolve to intersect Earth's orbit and produce meteor showers at Earth. Only few of those are known. Meteoroid streams that move in orbits with Tisserand parameter well in excess of 3 are the Geminids and Daytime Sextantids of the Phaethon complex and the lesser known epsilon Pegasids. The observed activity appears to be related to nearly whole scale disintegrations, rather than dust ejection from volatile outgassing as observed in active comets. There is only a small population of asteroids with a main-belt origin that recently disintegrated into meteoroid streams.
\end{abstract}

Keywords. meteors, meteoroids, comets: general, minor planets, asteroids

\section{Introduction}

Asteroids in the main asteroid belt are known to produce dust from an episodic cometlike outgassing of volatiles, from the incidental collision with smaller asteroids, or from occasional disruptions of rubble-pile asteroids by spin-up or other mechanisms (Jewitt et al., 2015). All these mechanisms could also act on the same asteroids after they have evolved into resonances and have been perturbed into more eccentric orbits that bring them close to Earth.

If these mechanisms do act on asteroids in the relatively short (1-100 My) timescale that they are Near Earth Asteroids (Bottke et al., 2002), then they would produce meteoroid streams, some of which will evolve to intersect Earth's orbit and manifest as our meteor showers. That makes meteor shower studies at Earth a tool to investigate the proposed mechanisms to turn asteroids into active asteroids and evaluate how important these mechanisms are to understand their mass loss.

\section{Meteoroid streams on high $T_{J} \gg 3$ orbits}

Very few meteor showers are known to move on orbits with a high Tisserand Parameter with respect to Jupiter $\left(T_{J} \gg 3\right)$. Figure 1 is a compilation of all meteoroid orbits detected by the Cameras for Allsky Meteor Surveillance (CAMS) project in California between October 21, 2010 and March 31, 2015 (Jenniskens et al., 2015a). Each point is one meteoroid orbit, displayed through out the year as a function of solar longitude $\left(\lambda_{0}\right)$, representing the position of Earth in its orbit. While many Taurid shower meteoroids have $T_{J} \sim 3.0-3.4$ (around $\lambda_{0}=200-300$ in Fig. 1), just into the asteroid domain, the only showers that have $T_{J}$ well in excess of 3 are the Geminids and Daytime Sextantids of the Phaethon complex (with $T_{J} \sim 4$.4) and the little known epsilon Pegasids (EPG, IAU\#326) with $T_{J} \sim 8$ (Fig. 1). Some other minor showers with high Tisserand parameter were detected in the Canadian Meteor Orbit Radar (CMOR) survey (Brown et al., 2008; 2010), but are not confirmed by CAMS (Jenniskens, 2015). Those could be showers 


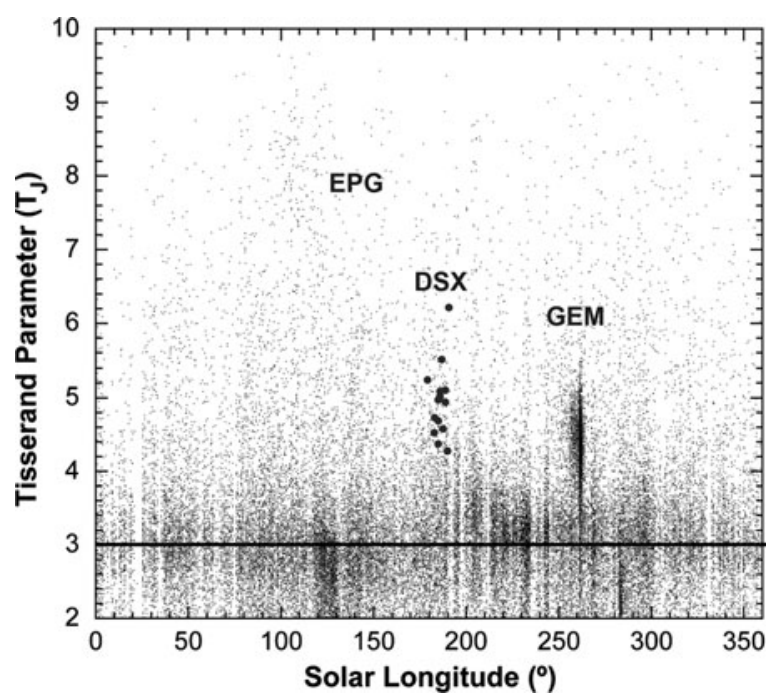

Figure 1. Tisserand parameter versus solar longitude of all CAMS-detected meteors between Oct. 21, 2010 and March 31, 2015. Thick dots mark the weakly detected Daytime Sextantids (DSX).

rich in faint meteors, because CMOR typically detects meteoroids ten times smaller in size than those detected by CAMS at a given speed (Jenniskens et al., 2015b).

\section{Geminids and Daytime Sextantids}

What we know about the Geminid meteor shower (GEM, \#4) from weakly active asteroid 3200 Phaethon is summarized in the review by Neslusan et al. (2015). The shower is significantly dispersed. CAMS detects the Geminid shower between about November 11 and December 22, in the period from solar longitude $\lambda_{0}=230-270^{\circ}$. CMOR has activity from the Geminids in the period $\lambda_{0}=225-282^{\circ}$ (Brown et al., 2010).

The shower's activity curve is asymmetric, rising slower than falling after the peak in the Earth's path. Some studies have detected a double-peaked structure at maximum on Dec. 12/13 (see the review in Jenniskens, 2006). There is a strong change in the particle size distribution, the most recent study of which is by Blaauw et al. (2011). This was interpreted as the result of a combined broader component rich in faint meteors and a narrow component more abundant in larger particles responsible for +2 and brighter meteors (Jenniskens, 2006).

The debris from 3200 Phaethon included large pebbles, up to $1 \mathrm{~kg}$ in mass, because the shower is a major source of lunar impacts (Suggs et al., 2014). It is not clear how such large grains are ejected from the parent body by water vapor drag in the mechanism proposed by Whipple (1950).

Early Geminids $\lambda_{0}<250^{\circ}$ are called theta Aurigids (THA, \#390). They have a shorter semi-major axis $a \sim 1.12 \mathrm{au}$, instead of the $a \sim 1.31$ au at the peak of the Geminid shower (Jenniskens et al., 2015a). There is a constant change of the value of longitude of perihelion along the path of the Earth.

The Geminid meteoroids behave different than many other meteoroids when entering Earth's atmosphere. They appear to have a relatively high meteoroid density of about 3 $\mathrm{g} / \mathrm{cm}^{3}$ (see discussion in Neslusan, 2015). Distinct flares are rarely observed. In regards 

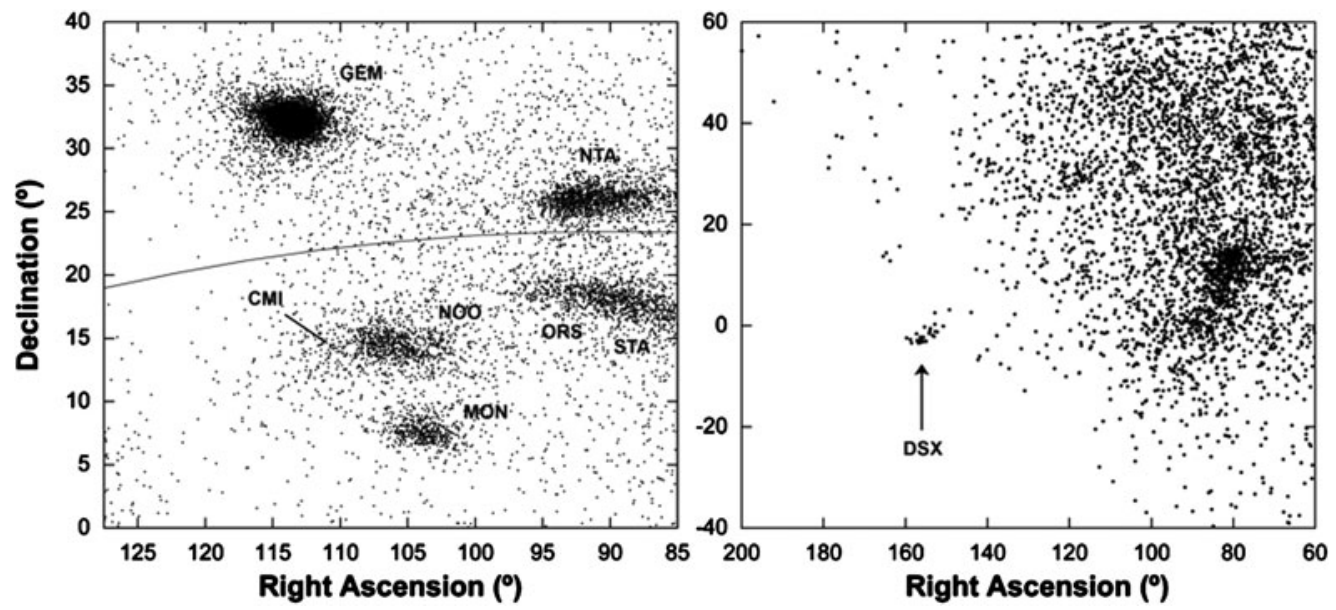

Figure 2. Drift-corrected geocentric radiant positions of the, left, Geminids (period solar longitude $\left.\lambda_{0}=230-271^{\circ}\right)$ and, right, the Daytime Sextantid Shower $\left(\lambda_{0}=182-191^{\circ}\right)$ in CAMS data (Jenniskens et al., 2015a). Meteor showers are labeled according to their IAU 3-letter shower code.

to their beginning height, they fall in group B of the scheme by Ceplecha (1988), thought to signify dense cometary material.

The Daytime Sextantids (DSX, \#221) move in an orbit as expected if the Geminids evolved for a period of about $4600 \mathrm{y}$ (Ohtsuka et al., 2006). The main result of that evolution is a rotation of the nodal line. In this evolution, asteroid Phaethon is still found close to its meteoroid stream, the Geminids, while parent body 2005 UD is still found close to the Daytime Sextantids stream. The ascending node of 3200 Phaethon is at $265.3^{\circ}$, while the Geminids at their peak have a node at $262.0^{\circ}$. Phaethon would evolve to the orbit of the Geminids in only about $220 \mathrm{y}$. Asteroid 2005 UD has a node at $19.8^{\circ}$, while the Daytime Sextantids have a node at $8.1^{\circ}$.

The December Canis Minorids (CMI, \#253) appear to be a twin shower of the Geminids just south of the ecliptic plane (Fig. 2) and may represent dust that has evolved sufficiently for the nodal line to rotate $180^{\circ}$. The median semi-major axis measured by CAMS is $a=1.88 \mathrm{au}$, as opposed to $1.271 \mathrm{au}$ for the current orbit of Phaethon (Jenniskens et al., 2015a).

The Geminids have a low perihelion distance $q \sim 0.14 \mathrm{au}$, which raises the question whether the activity of 3200 Phaethon may be related to this. Other meteor showers with low-q orbits are shown in Fig. 3. They include the Southern delta Aquariids (SDA, \#5) and the Northern Delta Aquariids (NDA, \#26) of the 96P/Machholz complex, the shortperiod Northern June Aquilids (NZC, \#164), the December Monocerotids (MON, \#19) from comet C/1917 F1 (Mellish), and the long-period and retrograde moving January Leonids (JLE, \#319).

Few meteoroid orbits have perihelion distance $q<0.04 \mathrm{au}$, where the meteoroids are expected to be heated to the point of sublimating the minerals (Figs. 3 and 4). The Geminids meteoroids also experience relatively high temperatures that can affect the thermophysical properties of the meteoroids (Capek and Borovicka, 2009), but they have never been much closer to the sun than $q \sim 0.14$ au. 


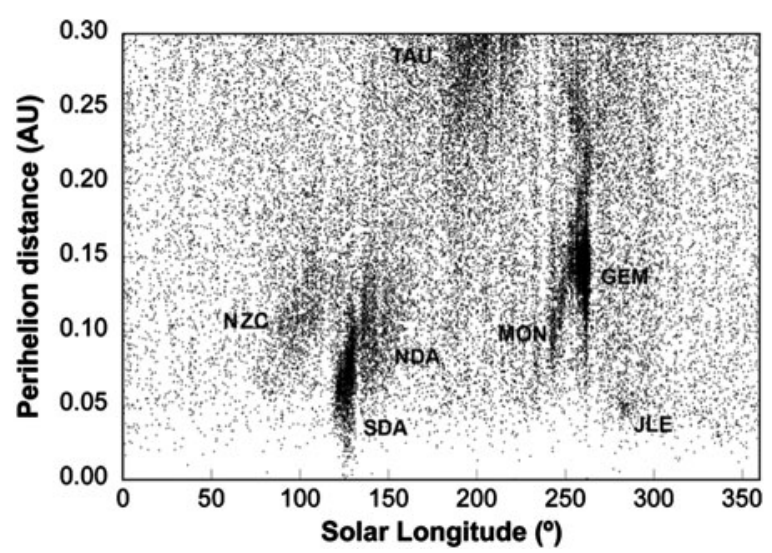

Figure 3. All meteoroids with $q<0.30$ au as a function of time in the year (solar longitude $\left.\lambda_{0}\right)$. The main showers are identified.

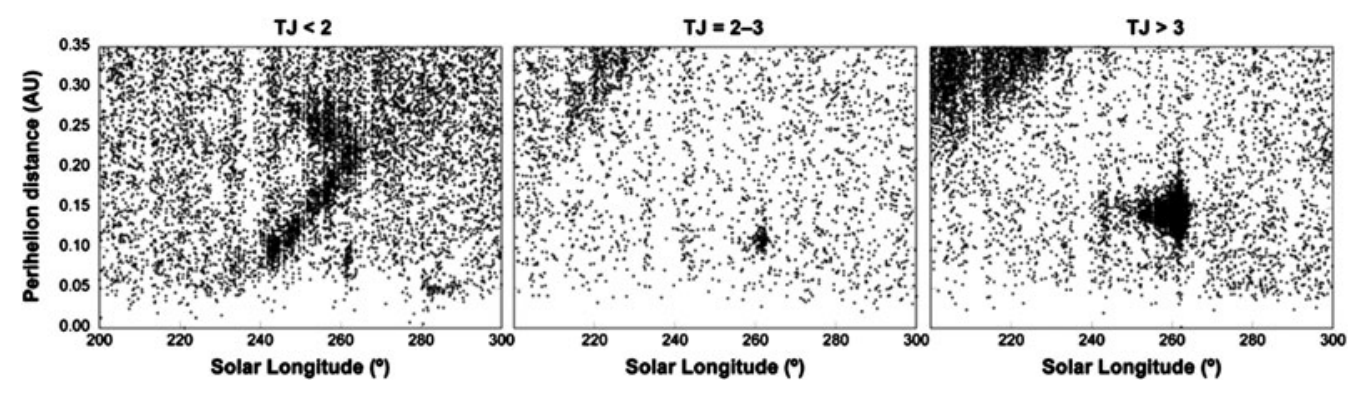

Figure 4. Detail of Fig. 3, separated in three intervals of Tisserand parameter. Low perihelion distance meteoroid orbits near the position of the Geminids.

\section{The epsilon Pegasids}

The epsilon Pegasids (EPG, \#326) were discovered in CMOR radar observations by Brown et al. (2008). Of several short-period streams picked up by CMOR, only this shower is recognized in CAMS data. The radiant is diffuse and stands out only weakly above the sporadic background. Fig. 5 shows the meteoroids isolated with a Discriminant algorithm (Jenniskens, 2015; Jenniskens et al., 2015a). The shower peaks on July $7\left(\lambda_{0}=\right.$ $105.5^{\circ}$ ) and has a diffuse radiant around R.A. $=326^{\circ}$, Decl. $=+15^{\circ}$ (Fig. 5) with entry speed $\mathrm{Vg}=30 \mathrm{~km} / \mathrm{s}$. The orbital elements are semi-major axis $a=0.757 \mathrm{au}$, perihelion distance $q=0.173 \mathrm{au}$, eccentricity $e=0.771$ and inclination $i=55.4^{\circ}$. No parent body is known.

Meteoroids in this stream have beginning heights in the lower group of meteoroids (Fig. 5). These were called group A in the classification scheme by Ceplecha (1988). These meteoroids have thermal properties that delay ablation until deeper in the atmosphere. In comparison, the Geminids (and delta Aquariids/Quadrantids) fall in between the high and low group (group B), while the Taurids of comet $2 \mathrm{P} /$ Encke are in the higher group C (Fig. 5).

\section{Discussion}

Is Phaethon an asteroid or a dormant Jupiter-family comet? The high Tisserand parameter suggests its origin is in the main belt. The reflection properties of the surface 

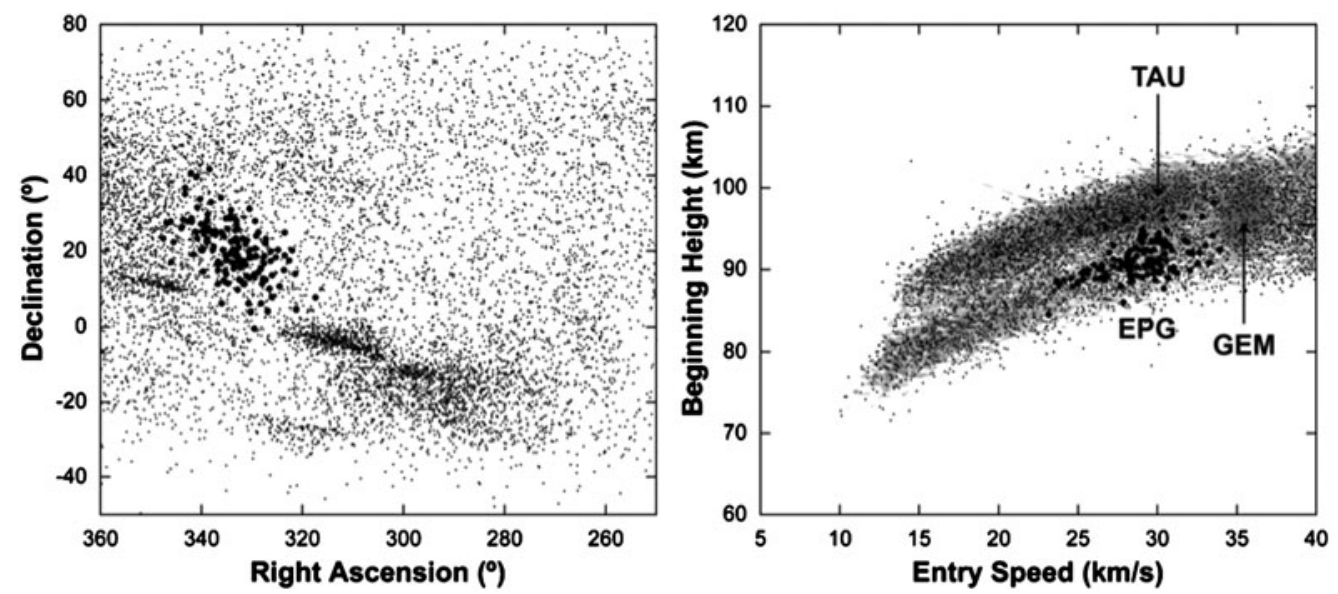

Figure 5. Radiant map and beginning heights of epsilon Pegasids (large dots) compared to other meteoroids detected in early July (small dots) in CAMS data during period $\lambda_{0}=94-118^{\circ}$. Also shown are beginning and end heights of other meteors observed in CAMS data, marking the Taurids (TAU) and Geminids (GEM).

and high inclination of the orbit point to an origin in the 2 Pallas asteroid family (de León et al., 2010; Jenniskens et al., 2010). The meteoroids have higher density that most cometary meteoroids observed at Earth, but the meteoroid density and thermal properties may have been affected by heating due to the low perihelion distance of the stream.

When did 3200 Phaethon arrive in the inner solar system? Based on the relative position along the precession cycle, it was only $10 \mathrm{ky}$ or so ago that $2005 \mathrm{UD}$ split from Phaethon, suggesting that the precursor body arrived relatively recently in the inner solar system, unlike in the dynamical models by de León et al. (2010). The object may have arrived from the 2 Pallas family rapidly via the 5:2 mean-motion resonance.

When was the Geminid stream created? Our best estimate is around the year 1030 $\mathrm{AD}$, based on the observed activity of the shower since it was first seen in the early 19th century (Jenniskens, 2006). Jones \& Hawkes (1986) demonstrated that the rotation of the nodal line causes the cluster of nodes of the stream to evolve around the circumference of the asteroid orbit. If created around $1030 \mathrm{AD}$, the shower will peak around $2050 \mathrm{AD}$, and be gone by $2100 \mathrm{AD}$. The Geminids are not recognized in medieval records.

Was the production of meteoroids sudden or the product of an accumulated period of activity over time? The total mass in the stream is about $28 \times 10^{12} \mathrm{~kg}$, about half of the $69 \times 10^{12} \mathrm{~kg}$ mass of 3200 Phaethon (Jenniskens, 2006, Table 8). The current activity of Phaethon is very low, not sufficient to explain the large mass in the stream (Jewitt et al., 2013). The activity appears to have been rather sudden as a result of a disruption, rather than normal cometary activity. However, there is currently no good meteoroid stream model that explains all features of the observed Geminid shower.

How is 2005 UD related to 3200 Phaethon? Long after splitting from Phaethon, 2005 UD itself created a significant amount of meteoroids. Hence, there appears to be an ongoing cascade of disruptions that are the main mass-loss mechanism for these objects. Both Phaethon and 2005 UD are still intimately associated with their meteoroid streams, which implies that the streams do not last long, significantly shorter than the time since Phaethon and 2005 UD broke apart. This phenomenon is also observed for other meteor shower parent body complexes that are thought to have a Jupiter-family origin, i.e. those associated with 96P/Machholz, 169P/NEAT, and 2P/Encke (Jenniskens et al., 2015a). 
Was the meteoroid stream the result from the first passage of the precursor object into the inner solar system? Neither Geminids nor Sextantids were from the original disruption that created Phaethon and 2005 UD. However, the December Canis Minorids may represent meteoroids that trace back longer in time. Either these meteoroids evolved more rapidly by an accelerated precession of their nodal line, or they were released a long time ago during breakup of the Phaethon/2005 UD progenitor.

What is the nature of the current weak activity of Phaethon and what will be the consequences? This is an interesting question. The current weak activity could be unrelated to what created the Geminid meteoroid stream, or be a precursor of future disruptions.

Is the Phaethon complex a unique case? The Geminids account for $15 \%$ of present day mass influx of mm- and cm-sized meteoroids still approaching Earth in the form of meteoroid streams (Jenniskens et al., 2015b). Only $2 \%$ of mass is contributed by sporadic meteors with $T_{J} \gg 3$ other than the Geminids. That said, it is important to realize that the Geminids are a transient shower at Earth. Activity will not last.

Do other disintegrations contribute to the large-grain sporadic population? There is a component of low-a orbits in the population of sporadic meteoroids, even for the cm-sized particles detected by CAMS (Jenniskens et al., 2015b). Given the lack of low-a streams, this component was interpreted to be the results of long periods of Poynting-Robertson drag, implying that collisional lifetimes are much longer than thought before and mmto cm-sized meteoroids must fade from the population by disintegration, rather than collisions (Jenniskens et al., 2015b).

The rapid disintegration of meteoroids by processes other than collisions is also seen for low albedo and relatively weak CM-type carbonaceous chondrites. CM chondrites as a group have cosmic ray exposure times of less than $2 \mathrm{Ma}$, short compared to other meteorite types. Sutter's Mill's cosmic ray exposure time was only 90000 y (Jenniskens et al., 2012). It is unlikely that a collisional breakup in the asteroid belt occurred this recently. The evolution of meteoroids into the resonances alone tends to take more time than that, while additional time is needed to evolve into an eccentric Earth crossing orbit. Both Sutter's Mill and Maribo fell to Earth from eccentric orbits with perihelion at $q \sim 0.3$ au (Jenniskens et al., 2012).

Are approaches to lower perihelion distance the trigger for asteroid disintegrations? Not often. Only the Phaethon complex and the epsilon Pegasids are known high $T_{J}$ asteroidal streams. There are not many more asteroidal streams from low q orbits (Jenniskens, 2015).

What is the mechanism responsible for the asteroidal streams? Ejection by volatiles does not appear to be an important mass-loss mechanism. Some primitive asteroids in the outer main belt are thought to be active because of volatile outgassing, the sublimation of ices dragging along meteoroids (Whipple, 1950). However, most of our near-Earth asteroids have their origin in the inner and middle parts of the main asteroid belt, arriving from the $\nu 6,3: 1$ or 5:2 mean-motion resonances (Bottke et al., 2002). When more primitive outer main belt objects arrive at the more distant resonances, their orbits will also become eccentric, but their aphelion will move into the path of Jupiter before their perihelion is low enough to cross Earth's orbit.

The cause of the disintegrations is an open question, but the mechanism of how to create large amounts of dust from asteroid disintegrations may be as follows. Asteroids are known to be rubble-pile objects, the smaller ones held together not by gravity but by the van der Waals forces from a cement of the smallest grains in the population of fragments (Scheeres et al., 2015). If such objects disintegrate, they could release copious amounts of meteoroids that can be detected as meteoroid streams at Earth. 


\section{Conclusions}

Disruptions of near Earth asteroids may have resulted in the Phaethon complex meteor showers (Geminids and Daytime Sextantids) and the epsilon Pegasids shower, among just a handful of other known streams. These showers do not seem to result from volatile outgassing, but from mechanisms that result in significant disintegration, where the parent body looses a significant fraction of mass. This type of disintegration is similar to that observed for Jupiter family comet showers that are parent bodies of other meteoroid streams. The meteoroid streams do not survive long, not because of dispersion of the dust or collisions, but because of the disintegration of the meteoroids by processes other than collisions.

The dispersion of dust in Earth's path provides strong constraints on dust formation epoch and conditions of ejection, but satisfactory models for the Geminid and Sextantid showers are still eluding us. The parent body of the epsilon Pegasids has not yet been identified.

\section{Acknowledgments}

This paper benefitted from a careful review by Jiri Borovicka. I would like to thank the amateur and professional astronomers who helped build and operate the CAMS network over the years. CAMS stations are hosted by Lick Observatory and Fremont Peak Observatory, and at private residences in Sunnyvale and Foresthill, California. This work is supported by the NASA NEOO program.

\section{References}

Blaauw, R. C., Campbell-Brown, M. D., \& Weryk, R. J. 2011, Month. Not. Roy. Astron. Soc., 414,3322

Bottke, W. J., Morbidelli, A., Jedicke, R., Petit, J.-M., Levison, H. F., Michel, P., \& Metcalfe, T. S. 2002, Icarus, 156, 399

Brown, P., Weryk, R. J., Wong, D. K., \& Jones, J. 2008, Icarus, 195, 317

Brown, P., Wong, D. K., Weryk, R. J., \& Wiegert, P. 2010, Icarus, 207, 66

Capek, D. \& Borovicka, J. 2009, Icarus, 202, 361

Ceplecha, Z 1988, Bull. Astron. Inst. Czechosl., 39, 221

de León, J., Campins, H., Tsiganis, K., Morbidelli, A., \& Licandro, J. 2010, Astron. Astrophys., 513, 26

Jenniskens, P. 2006, Meteor Showers and their Parent Comets, Cambridge University Press, Cambridge, U. K., 790 pp.

Jenniskens, P. 2008, Icarus, 194, 13

Jenniskens, P., Vaubaillon, J., Binzel, R. P., DeMeo, F. E., Nesvorny, D., Bottke, W. F., Fitzsimmons, A., Hiroi, T., Marchis, F., Bishop, J. L., Vernazza, P., Zolensky, M. E., Herrin, J. S., Welten, K. C., Meier, M. M. M., \& Shaddad, M. H. 2010, Meteorit. Plan. Sci., 45, 1590

Jenniskens, P., and 69 colleagues 2012, Science, 338, 1583

Jenniskens, P. 2015, In: Asteroids IV. University of Arizona Space Science Series, P. Michel, F. DeMeo, W. Bottke, eds. p. 281

Jenniskens, P., Nénon, Q., Albers, J., Gural, P. S., Haberman, B., Holman, D., Grigsby, B. J., Samuels, D., \& Johannink, C. 2015a, The established meteor showers as observed by CAMS. Icarus, 266, 331

Jenniskens, P., Nénon, Q., Gural, P. S., Albers, J., Haberman, B., Johnson, B., Morales, R., Grigsby, B. J., Samuels, D., \& Johannink, C. 2015b, CAMS newly detected meteor showers and the sporadic background. Icarus, 266, 384

Jewitt D., Li, J. \& Agarwal, J. 2015, Astrophys. J., 771, L36

Jewitt D., Hsieh, H. \& Agarwal, J. 2015, In: Asteroids IV. University of Arizona Space Science Series, P. Michel, F. DeMeo, W. Bottke, eds. p. 222 
Neslusan, L. 2015, Contr. Astron. Obs. Skalnate Pleso 45, 60

Scheeres, D. J. 2015, Icarus, 247, 1

Jones, J. \& Hawkes, R. L. 1986, Month. Not. Roy. Astron. Soc., 223, 479

Ohtsuka, K., Sekiguchi, T., Kinoshita, D., Watanabe, J.-I., Ito, T., Arakida, H., \& Kasuga, T. 2006, Astronomy and Astrophysics, 450, L25

Suggs, R. M., Moser, D. E., Cooke, W. J., \& Suggs, R. J. 2014, Icarus, 238, 23

Whipple, F. L. 1950, The Astrophysical Journal, 111, 375 